\title{
Variational theory combining number-projected BCS and coupled-cluster doubles
}

\author{
V. V. Baran $\oplus^{1,2, *}$ and J. Dukelsky $\oplus^{3, \dagger}$ \\ ${ }^{1}$ Faculty of Physics, University of Bucharest, 405 Atomiştilor, POB MG-11, Bucharest-Măgurele, RO-077125, Romania \\ 2 "Horia Hulubei” National Institute of Physics and Nuclear Engineering, 30 Reactorului, RO-077125, Bucharest-Măgurele, Romania \\ ${ }^{3}$ Instituto de Estructura de la Materia, CSIC, Serrano 123, E-28006 Madrid, Spain
}

(Received 22 February 2021; accepted 6 May 2021; published 24 May 2021)

\begin{abstract}
The ground-state pairing correlations in finite fermionic systems are described with a high degree of accuracy within a variational approach based on a combined coupled-cluster and particle-number-projected BCS ansatz. The flexibility of this symmetry-preserving wave function enables a unified picture valid from weak to strong coupling, both in small and large systems. The present variational approach consistently yields an energy upper bound while operating at the same level of precision as the nonvariational particle-number-projected Bogoliubovcoupled-cluster theory [Phys. Rev. C 99, 044301 (2019)].
\end{abstract}

DOI: 10.1103/PhysRevC.103.054317

\section{INTRODUCTION}

Pairing Hamiltonians are ubiquitous in quantum manybody physics. Starting from their variational treatment in the microscopic theory of superconductivity given by Bardeen, Cooper and Schrieffer (BCS) [1], they were soon exported to nuclear physics for the description of the large gaps observed in even-even nuclei [2]. However, the violation of particle number in the BCS theory, which is negligible for macroscopic systems, represents a major drawback when applied to finite systems. Therefore, techniques to implement number projection on top of the BCS wave function (PBCS) were developed in nuclear structure $[3,4]$ and more recently in quantum chemistry [5,6] where the PBCS wave function is known as the antisymmetrized geminal power (AGP). PBCS improves over the BCS theory in finite systems, especially in the strong coupling limit where superconductivity is well established, but it still fails in the weak coupling limit dominated by pairing fluctuations, and all along the transitional region $[7,8]$. This fact was made evident in ultrasmall superconducting grains, where PBCS predicted an abrupt metal-superconductor transition as a function of the grain size [9] while the exact solution showed a smooth crossover dominated by large fluctuations [10]. It was precisely in the field of ultrasmall superconducting grains that the exact solution of the constant pairing Hamiltonian given by Richardson in the sixties [11] was recovered [12] and intensively used as a natural benchmark model for superconducting theories beyond BCS [13-21].

In the extreme weak coupling limit pair coupled-cluster doubles (pCCD) describes correctly the pairing fluctuating regime but it quickly overbinds because of the nonvariational character of the theory based on the left projection to a Hartree-Fock Slater determinant [8,14,22]. It seems, therefore, that a combination of pCCD and quasiparticle BCS

\footnotetext{
*vvbaran@fizica.unibuc.ro

$\dagger$ j.dukelsky@csic.es
}

would be able to approach both limits correctly. Indeed, the extension of pCCD to BCS quasiparticles, the BCS-CCD method [15], gave the correct behavior in the weak coupling limit, but still suffers from large deviations $(\sim 10 \%$ for sizes of $\sim 100$ particles) across the transition region. There were attempts to interpolate between pCCD and PBCS $[8,16]$ or to diagonalize the pairing Hamiltonian in a subspace defined by the reference PBCS state and different two and four numberprojected quasiparticle states [23]. Other possible ways to add correlations to the PBCS state were explored in [21,24]. A different, and perhaps more sophisticated approach, is based on the use of the Richardson ansatz as a reference state for variational or configuration interaction approximations in nonintegrable pairing or spin models $[25,26]$.

Perhaps, the most successful theory beyond BCS-CCD is simply its number-projected version coined as particlenumber-projected BCS coupled-cluster doubles (PBCS-CCD) theory [27]. The theory is not Ritz variational and therefore, it cannot assure an upper bound for the ground-state energy. However, it has an affordable computational cost that scales polynomially with the system size. Moreover, it gives excellent numerical results both in the weak and strong coupling limits, as well as in the transitional region.

The aim of our paper is to design a Ritz variational method with a trial wave function that combines PCCD and PBCS and produces numerical results with the same level of precision of PBCS-CCD and with a similar computational cost. We will benchmark our variational theory with the exact solution of the Richardson model [28] and with PBCS-CCD results [27] where available.

\section{THEORETICAL BACKGROUND}

\section{A. Pairing Hamiltonian}

We consider the generic pairing Hamiltonian,

$$
H=\sum_{i=1}^{L} \epsilon_{i}\left(c_{i}^{\dagger} c_{i}+c_{\bar{i}}^{\dagger} c_{\bar{i}}\right)+\sum_{i, j=1}^{L} V_{i, j} c_{i}^{\dagger} c_{\bar{i}}^{\dagger} c_{\bar{j}} c_{j},
$$


where $i$ and $\bar{i}$ indicate one of the $L$ pairs of conjugated degenerate single-particle levels with energy $\epsilon_{i}=\epsilon_{\bar{i}}$.

The Hamiltonian (1) preserves seniority, and for simplicity we restrict ourselves to the seniority zero $(v=0)$ subspace. Then, in the absence of the interaction term the ground state is given by the Hartree-Fock product state,

$$
|\mathrm{HF}\rangle=\prod_{i=1}^{M} c_{i}^{\dagger} c_{\bar{i}}^{\dagger}|0\rangle,
$$

where $M$ is the number of pairs in the system.

In preparation for the discussion that follows, we pass to the particle-hole $(\mathrm{ph})$ representation and denote the particle levels with $p>M$ and the hole levels with $h \leqslant M$. We define the particle and hole pair and number operators,

$$
\begin{aligned}
& P_{p}^{\dagger}=c_{p}^{\dagger} c_{\bar{p}}^{\dagger}, P_{p}=c_{\bar{p}} c_{p}, N_{p}=c_{p}^{\dagger} c_{p}+c_{\bar{p}}^{\dagger} c_{\bar{p}}, \\
& P_{h}^{\dagger}=c_{\bar{h}} c_{h}, P_{h}=c_{h}^{\dagger} c_{\bar{h}}^{\dagger}, N_{h}=2-c_{h}^{\dagger} c_{h}-c_{\bar{h}}^{\dagger} c_{\bar{h}},
\end{aligned}
$$

such that the Hartree-Fock state (2) is the vacuum to the $P$ and $N$ operators, $P_{p}|\mathrm{HF}\rangle=P_{h}|\mathrm{HF}\rangle=0, N_{p}|\mathrm{HF}\rangle=N_{h}|\mathrm{HF}\rangle=$ 0 . The pairing Hamiltonian (1) is then expressed as

$$
\begin{aligned}
H= & E_{\mathrm{HF}}+\sum_{p=M+1}^{L} \epsilon_{p} N_{p}+\sum_{h=1}^{M}\left(-\epsilon_{h}-V_{h h}\right) N_{h} \\
& +\sum_{p, p^{\prime}=M+1}^{L} V_{p p^{\prime}} P_{p}^{\dagger} P_{p^{\prime}}+\sum_{h, h^{\prime}=1}^{M} V_{h h^{\prime}} P_{h}^{\dagger} P_{h^{\prime}} \\
& +\sum_{p=M+1}^{L} \sum_{h=1}^{M} V_{p h}\left(P_{p}^{\dagger} P_{h}^{\dagger}+P_{h} P_{p}\right),
\end{aligned}
$$

with $E_{\mathrm{HF}}=\langle\mathrm{HF}|H| \mathrm{HF}\rangle=\sum_{h=1}^{M}\left(2 \epsilon_{h}+V_{h h}\right)$ being the energy of the Hartree-Fock state (2).

\section{B. Mean-field theory and its symmetry restoration}

The standard description of the pairing correlations induced by the Hamiltonian (1) is given within the BCS approximation [1] in terms of the pair condensate,

$$
|\mathrm{BCS}\rangle=\exp \left[\Gamma^{\dagger}(x)\right]|0\rangle, \quad \Gamma^{\dagger}(x) \equiv \sum_{i=1}^{L} x_{i} c_{i}^{\dagger} c_{\bar{i}}^{\dagger},
$$

which explicitly breaks the $U(1)$ gauge symmetry associated with particle number conservation. For macroscopic systems the symmetry broken picture is exact and the particle number fluctuations are negligible. However, for finite systems like atomic nuclei or small superconducting grains one speaks only of obscured or emergent symmetry breaking [29-32], in which case the quantum fluctuations inevitably lift any degeneracy associated with the broken symmetry.

Much effort is thus devoted to restore the symmetry of the mean-field ansatz with the help of projection techniques [33-39]. In the BCS case upon particle number restoration we obtain the so-called number-projected BCS (PBCS) [3] or antisymmetrized geminal power (AGP) in the context of quantum chemistry [40],

$$
\begin{aligned}
|\operatorname{PBCS}(x)\rangle & =\mathcal{P}_{M}|\mathrm{BCS}\rangle \\
& =\frac{1}{M !}\left[\Gamma^{\dagger}(x)\right]^{M}|0\rangle \\
& =\frac{1}{2 \pi} \int_{0}^{2 \pi} \mathrm{d} \theta e^{-i \theta M} \exp \left[\Gamma^{\dagger}\left(e^{i \theta} x\right)\right],
\end{aligned}
$$

where $\mathcal{P}_{M}$ is the projector onto the state of $M$ pairs. While PBCS describes well the properties of superfluid nuclei with a small number of valence nucleons [36], it cannot account for the weak pairing correlations that develop within larger spaces, e.g., those considered in the large-scale energy density functional treatments of finite nuclei or in the study of small superconducting grains [10]. For a working description of the weak pairing regime one usually turns to RPA [14] or coupled-cluster $[8,15,22]$ approaches. Generalizations of the PBCS ansatz have also been considered based on its structural similarity with a particular coupled-cluster ansatz [8]. Specifically, the PBCS representation in the ph basis is obtained as

$$
\begin{aligned}
|\mathrm{PBCS}\rangle & \propto \sum_{\ell=0}^{M} \frac{1}{\ell !^{2}}\left[\Gamma_{P}^{\dagger}(x) \Gamma_{H}^{\dagger}(1 / x)\right]^{\ell}|\mathrm{HF}\rangle \\
& =\frac{1}{2 \pi} \int_{0}^{2 \pi} \mathrm{d} \theta \exp \left[\Gamma_{P}^{\dagger}\left(e^{i \theta} x\right)\right] \exp \left[\Gamma_{H}^{\dagger}\left(e^{-i \theta} / x\right)\right]|\mathrm{HF}\rangle,
\end{aligned}
$$

in terms of the particle and hole components of the collective pairs,

$$
\Gamma_{P}^{\dagger}(x)=\sum_{p=M+1}^{L} x_{p} P_{p}^{\dagger}, \quad \Gamma_{H}^{\dagger}(x)=\sum_{h=1}^{M} x_{h} P_{h}^{\dagger} .
$$

The structure of the PBCS state is then defined by the inverse squared factorials appearing as expansion coefficients in the collective ph-pair basis.

\section{Coupled-cluster theory}

Analogously, by using a slightly modified expansion involving plain factorials one obtains a separable pair coupledcluster doubles variational ansatz $\left(\mathrm{vCCD}_{\mathrm{sep}}\right)$,

$$
\begin{aligned}
\left|\mathrm{vCCD}_{\mathrm{sep}}\right\rangle & =\sum_{\ell=0}^{M} \frac{1}{\ell !}\left[\Gamma_{P}^{\dagger}(y) \Gamma_{H}^{\dagger}(y)\right]^{\ell}|\mathrm{HF}\rangle \\
& =\exp \left[\Gamma_{P}^{\dagger}(y) \Gamma_{H}^{\dagger}(y)\right]|\mathrm{HF}\rangle \\
& =\exp \left[\sum_{p, h} y_{p} y_{h} P_{p}^{\dagger} P_{h}^{\dagger}\right]|\mathrm{HF}\rangle .
\end{aligned}
$$

This is a particular case of a pair coupled-cluster doubles variational wave function (vCCD),

$$
|\mathrm{vCCD}\rangle=\exp \left[\sum_{p, h} z_{p h} P_{p}^{\dagger} P_{h}^{\dagger}\right]|\mathrm{HF}\rangle,
$$

involving the most general double excitations that do not break pairs through a fully nonseparable structure matrix 


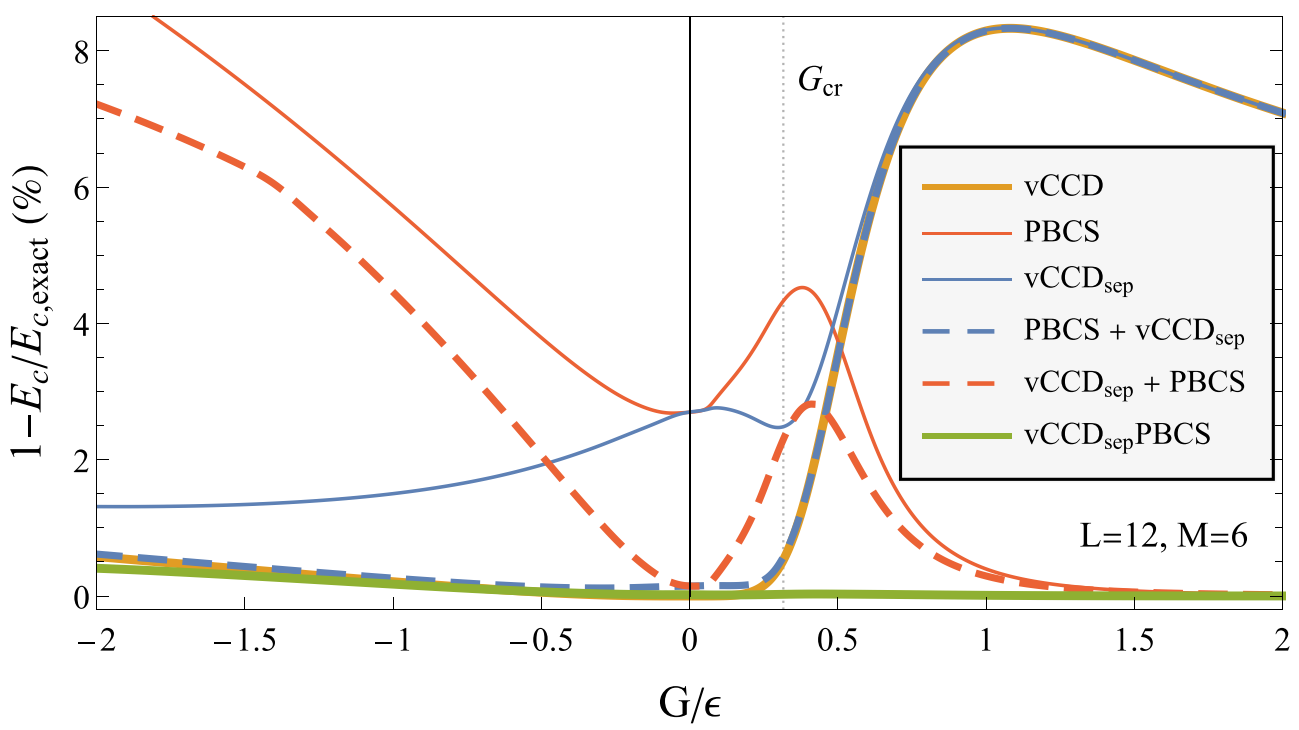

FIG. 1. Error in the correlation energy (29) relative to its exact value (in percentages) versus the pairing strength $G$ (in units of the level spacing $\epsilon$ ) for the fully variational vCCD (10), PBCS (6), and $\mathrm{vCCD}_{\text {sep }}$ PBCS (13) wave functions in the case of an $L=12$ level system at half filling. The "vCCD ${ }_{\text {sep }}+\mathrm{PBCS}$ " procedure indicated by a red dashed line involves an energy minimization with respect to the vCCD amplitudes in the presence of the fixed optimal PBCS reference. Within the "PBCS+ $\mathrm{vCCD}_{\text {sep }}$ " procedure indicated by a blue dashed line the PBCS amplitudes are varied in the presence of the fixed optimal $\mathrm{vCCD}_{\text {sep }}$ structure.

$z_{p h}[14]$. The separable case $\mathrm{vCCD}_{\text {sep }}$ is thus recovered for $z_{p h}=y_{p} y_{h}$.

On the one hand, the full freedom in the structure matrix of the CCD ansatz allows for an excellent description of the weak pairing regime where the exact solution to leading order involves the nonseparable pair-excitation amplitudes $z_{p h}=$ $(G / 2) /\left(\epsilon_{p}-\epsilon_{h}\right)$. This is from the additional beyond-pair correlations (in the sense of Ref. [8]) accounted for relative to the separable case. Note, for example, that the Richardson solution involving complex-conjugated pairs may be expressed exactly as a product of four-body quartet structures [41]. On the other hand, the computational complexity of evaluating operator matrix elements exactly in the nonseparable case grows exponentially with the size of the system. The usual approximation of coupled cluster involves a left projection onto the subspaces of zero and two ph pairs, thus breaking the Ritz variational principle. Furthermore, even with a fully nonseparable structure matrix, the validity of the vCCD ansatz breaks down around the critical value of the pairing strength (see also Fig. 1). The choice of a BCS mean-field reference state does improve on this aspect at the cost of effectively breaking the particle number symmetry [15].

Given the success of vCCD in the weak pairing regime and that of PBCS in the strong pairing regime, it is then natural to combine them for a precise unified description of all regimes.

\section{Combining coupled-cluster and symmetry-restored mean-field theories}

The symmetry restoration of broken-symmetry coupledcluster theories was only recently considered [27,42-44]. For the schematic pairing Hamiltonian with the breaking and restoration of the particle-number symmetry, the so-called particle-number-projected Bogoliubov-coupled-cluster theory yields highly accurate results [18]. In this context, a set of differential equations is set up for obtaining the gaugeangle-dependent excitation operator. The practical need for truncating this set of equations implies, however, an approximate action of the projection operator, which together with the truncation of the cluster excitation operator itself leads to a violation of the Ritz variational principle.

It is the purpose of this work to explore a physically transparent alternative in the form of a variational CCD-PBCS combined approach. Ideally the ground state would involve the CCD excitations built directly on top of the particlenumber-projected BCS as

$$
|\mathrm{vCCD}-\mathrm{PBCS}\rangle=\exp \left[\sum_{p, h} z_{p h} P_{p}^{\dagger} P_{h}^{\dagger}\right]|\operatorname{PBCS}(x)\rangle,
$$

where the $L$ mixing amplitudes $x_{i}$ of the PBCS state and the $M(L-M)$ structure matrix elements $z_{p h}$ of the CCD excitations are to be treated as free variational parameters. Indeed, the energy minimization procedure,

$$
E_{\mathrm{gs}}=\min _{\psi} \frac{\langle\psi|H| \psi\rangle}{\langle\psi \mid \psi\rangle},
$$

yields extremely precise results for the few small systems where this ansatz may actually be applied.

Computational access to larger systems is enabled upon various simplifications. Let us consider first the separability approximation $z_{p h}=y_{p} y_{h}$ for the structure matrix of the CCD excitations in Eq. (11), yielding the $\mathrm{vCCD}_{\text {sep }}$ PBCS ansatz,

$$
\left|\mathrm{vCCD}_{\text {sep }} \mathrm{PBCS}\right\rangle=\exp \left[\Gamma_{P}^{\dagger}(y) \Gamma_{H}^{\dagger}(y)\right]|\operatorname{PBCS}(x)\rangle,
$$

which involves $2 L$ independent variational parameters $x_{i}, y_{i}$. Remarkably, while the PBCS ansatz (6) and the $\mathrm{vCCD}_{\text {sep }}$ 
ansatz (9) each individually fail in the weak pairing regime because of their separable structure matrices, the combined $\mathrm{vCCD}_{\text {sep }}$ PBCS wave function will turn out to be quite accurate. This may be easily understood by noting that for weak pairing the above ansatz effectively involves a nonseparable structure matrix in the form,

$$
\left|\mathrm{vCCD}_{\mathrm{sep}} \mathrm{PBCS}\right\rangle \approx\left[1+\sum_{p, h}\left(x_{p} x_{h}+y_{p} y_{h}\right) P_{p}^{\dagger} P_{h}^{\dagger}\right]|\mathrm{HF}\rangle,
$$

obtained after taking into account the PBCS (6) and vCCD sep (9) expansions and redefining $x_{h} \rightarrow 1 / x_{h}$ in (7). The accuracy of the doubly separable $2 L$-dimensional parametrization of the fully nonseparable $M(L-M)$-dimensional structure matrix naturally degrades with increasing system size, the actual rate being numerically determined in the next section.

An alternative approximation scheme would then involve a fully nonseparable CCD excitation, limited, however, to a relatively small finite window around the Fermi level. This is from the exponential increase with the system size of the computational cost for evaluating the various matrix elements on the CCD wave function; see, e.g., Eqs. (26)-(28) below. We thus consider the combination,

$$
\left|\operatorname{vCCD}^{(w)} \operatorname{PBCS}(x, z)\right\rangle=\operatorname{vCCD}^{(w)}(z)|\operatorname{PBCS}(x)\rangle,
$$

with

$$
\operatorname{vCCD}^{(w)}(z)=\exp \left[\sum_{p=M+1}^{M+w / 2} \sum_{h=M+1-w / 2}^{M} z_{p h} P_{p}^{\dagger} P_{h}^{\dagger}\right],
$$

where $w$ denotes the size of the truncation window. As will be detailed in the next section, the quality of the results obtained within this approach will turn out to be inferior to that of the above vCCD ${ }_{\text {sep }}$ PBCS ansatz of Eq. (13) involving two sets of global parameters.

Overall the optimal compromise between the computational complexity and the accuracy of the results is found by combining the above two approximation schemes (9) and (15) into the ansatz,

$$
\begin{aligned}
\left|\operatorname{vCCD}_{\text {sep }}^{(w)} \operatorname{PBCS}(x, y, z)\right\rangle & =\operatorname{vCCD}_{\text {sep }}^{(w)}(y, z)|\operatorname{PBCS}(x)\rangle \\
\operatorname{vCCD}_{\text {sep }}^{(w)} & =\operatorname{vCCD}^{(w)}(z) \operatorname{vCCD}_{\text {sep }}(y)
\end{aligned}
$$

involving a total of $2 L+(w / 2)^{2}$ free variational parameters and leading to highly accurate results comparable to those of Ref. [18], to be discussed later on. Next, we shortly review the actual computational strategy for the above mentioned wave functions.

\section{E. Computational aspects}

In this section we propose a novel efficient algorithm for the evaluation of expectation values on the combined $\mathrm{vCCD}_{\text {sep }}^{(w)} \mathrm{PBCS}$ wave function (17). It is based on a representation of the PBCS (7), vCCD sep (19), and $\operatorname{vCCD}^{(w)}(16)$ terms as disentangled particle and hole gauge-angle-rotated BCS states.

We start from the discrete exact representation for the particle-number projection operation and decompose the
PBCS ansatz using the definitions of Eqs. (7) and (8):

$$
|\mathrm{PBCS}\rangle=\sum_{n=0}^{L} \exp \left[\Gamma_{H}^{\dagger}\left(e^{-i \theta_{n}} x\right)\right] \exp \left[\Gamma_{P}^{\dagger}\left(e^{i \theta_{n}} x\right)\right]|\mathrm{HF}\rangle,
$$

where $\theta_{n}=2 \pi n /(L+1)$. Note that we neglect the irrelevant constant normalization factors throughout this section.

The collective pairs (for the particle and hole subspaces) appearing in the $\mathrm{vCCD}_{\text {sep }}$ operator,

$$
\operatorname{vCCD}_{\text {sep }}=\sum_{\ell=0}^{M} \frac{1}{\ell !}\left[\Gamma_{P}^{\dagger}(y) \Gamma_{H}^{\dagger}(y)\right]^{\ell},
$$

are also expanded as superpositions of gauge-angle-rotated BCS operators,

$$
\begin{aligned}
& \frac{\left[\Gamma_{P}^{\dagger}(y)\right]^{\ell}}{\ell !}=\sum_{k=0}^{L-M} e^{-i \phi_{k} \ell} \exp \left[\Gamma_{P}^{\dagger}\left(e^{i \phi_{k}} y\right)\right], \\
& \frac{\left[\Gamma_{H}^{\dagger}(y)\right]^{\ell}}{\ell !}=\sum_{k=0}^{M} e^{-i \varphi_{k} \ell} \exp \left[\Gamma_{H}^{\dagger}\left(e^{i \varphi_{k}} y\right)\right],
\end{aligned}
$$

with $\phi_{k}=2 \pi k /(L-M+1)$ and $\varphi_{k}=2 \pi k /(M+1)$. Finally we obtain the representation,

$$
\begin{aligned}
\left|\mathrm{vCCD}_{\mathrm{sep}} \mathrm{PBCS}\right\rangle= & \sum_{k_{p}=0}^{L-M} \sum_{k_{h}=0}^{M} \sum_{n=0}^{L} g_{k_{p} k_{h}} \\
& \times \mathrm{BCS}_{P}\left(k_{p}, n\right) \mathrm{BCS}_{H}\left(k_{h}, n\right)|\mathrm{HF}\rangle,
\end{aligned}
$$

in terms of the gauge-angle-rotated BCS operators,

$$
\begin{aligned}
\operatorname{BCS}_{P}\left(k_{p}, n\right) & =\exp \left[\Gamma_{P}^{\dagger}\left(e^{i \theta_{n}} x+e^{i \phi_{k_{p}}} y\right)\right], \\
\operatorname{BCS}_{H}\left(k_{h}, n\right) & =\exp \left[\Gamma_{H}^{\dagger}\left(e^{-i \theta_{n}} x+e^{i \phi_{k_{p}}} y\right)\right],
\end{aligned}
$$

with expansion coefficients,

$$
g_{k_{p} k_{h}}=\sum_{\ell=0}^{M} \ell ! \exp \left[-i\left(\phi_{k_{p}}+\varphi_{k_{h}}\right) \ell\right] .
$$

We are interested in the expectation values of generic particle-number-conserving ph-factorized operators $\mathcal{O}=$ $\mathcal{O}^{(P)} \mathcal{O}^{(H)}$ on the vCCD ${ }_{\text {sep }}$ PBCS state (21). As the particlenumber projection operation needs only to be performed once on the mean-field BCS wave function, we obtain

$$
\begin{aligned}
\langle\mathcal{O}\rangle & =\left\langle\operatorname{vCCD}_{\mathrm{sep}} \mathrm{BCS}\left|\mathcal{O}^{(P)} \mathcal{O}^{(H)}\right| \mathrm{vCCD}_{\mathrm{sep}} \mathrm{PBCS}\right\rangle \\
& =\sum_{k_{p}, k_{p}^{\prime}=0}^{L-M} \sum_{k_{h}, k_{h}^{\prime}=0}^{M} g_{k_{p} k_{h}}^{*} g_{k_{p}^{\prime} k_{h}^{\prime}} \sum_{n=0}^{L} O_{k_{p}, k_{p}^{\prime}, n}^{(P)} \mathcal{O}_{k_{h}, k_{h}^{\prime}, n}^{(H)},
\end{aligned}
$$

involving the matrix elements between particle and hole BCS states (22),

$$
\begin{aligned}
O_{k_{p}, k_{p}^{\prime}, n}^{(P)} & =\left\langle\mathrm{BCS}_{P}\left(k_{p}, 0\right)\left|O^{(P)}\right| \mathrm{BCS}_{P}\left(k_{p}^{\prime}, n\right)\right\rangle, \\
\mathcal{O}_{k_{h}, k_{h}^{\prime}, n}^{(H)} & =\left\langle\mathrm{BCS}_{H}\left(k_{h}, 0\right)\left|O^{(H)}\right| \mathrm{BCS}_{H}\left(k_{h}^{\prime}, n\right)\right\rangle .
\end{aligned}
$$

By considering all terms in Eq. (4), the expression (24) may be directly employed to compute the energy expectation value $E=\langle H\rangle /\langle I\rangle$ on the $\mathrm{vCCD}_{\text {sep }} \mathrm{PBCS}$ wave function. 
To include the effects of the nonseparable $\operatorname{vCCD}^{(w)}$ excitations of Eq. (17) we use a Hubbard-Stratonovich transformation [45] and pass to a disentangled BCS representation of the vCCD operator,

$$
\begin{aligned}
\operatorname{vCCD}(z)= & \exp \left(\sum_{p, h} z_{p h} P_{p}^{\dagger} P_{h}^{\dagger}\right) \\
= & \int d^{L} \xi \exp \left(-\frac{1}{2} \xi^{T} Z^{-1} \xi\right) \\
& \times \exp \left[\Gamma_{P}^{\dagger}(\xi)+\Gamma_{H}^{\dagger}(\xi)\right],
\end{aligned}
$$

with

$$
Z=\left(\begin{array}{cc}
0 & z \\
z^{T} & 0
\end{array}\right)
$$

This BCS representation allows us to generalize the above Eq. (24) to the expectation values of ph-factorized operators on the $\mathrm{vCCD}_{\mathrm{sep}}^{(w)} \mathrm{PBCS}$ wave function (17). In practice the matrix elements on the resulting BCS wave functions which generalize Eq. (25) are to be treated as polynomials in the integration variables $\xi$. Only specific terms corresponding to the various nonzero Wick contractions are to be selected according to

$$
\int d^{L} \xi \exp \left(-\frac{1}{2} \xi^{T} Z^{-1} \xi\right) \xi_{i} \xi_{j} \ldots \xi_{k} \xi_{l}=\sum_{\text {Wick }} Z_{a b} \ldots Z_{c d}
$$

the set of indices $\{a, b, \ldots, c, d\}$ representing a permutation of $\{i, j, \ldots, k, l\}$ [46].

With the nonseparable CCD excitations restricted to a small window of $w$ levels around the Fermi level as in Eq. (15), the matrix elements of the relevant operators on generic $\mathrm{vCCD}^{(w)} \mathrm{BCS}$ states may be computed analytically (the notebook in CADABRA2 [47] is available upon request from the authors). Their subsequent coupling to the separable subspace (i.e., the complement of the $w$-level window) leads to the final $\mathrm{vCCD}_{\text {sep }}^{(w)} \mathrm{PBCS}$ results presented in the next section.

\section{NUMERICAL RESULTS}

In this section we benchmark the variational calculations for the various wave functions presented above against the exact solution [28] for a constant pairing Hamiltonian $\left[V_{i, j}=\right.$ $-G$ in Eq. (1)] of equally spaced single-particle levels with energies $\epsilon_{k}=k \epsilon, k=1, \ldots, L$. To avoid the singularities in the equations that solve exactly the picket fence model we use the same algorithm proposed by Richardson in [28].

The numerical code used to compute the expectation value of the Hamiltonian (4) on the various derivatives of the vCCDPBCS wave function is freely available upon request from the authors. The minimization procedure for the energy function (12) is performed using the e04ucf routine of the NAG library [48].

We present in Fig. (1) the errors for the correlation energy,

$$
E_{c}=\frac{\langle\psi|H| \psi\rangle}{\langle\psi \mid \psi\rangle}-E_{\mathrm{HF}},
$$

relative to its exact value for $L=12$ at half filling. On the one hand, notice how PBCS (6) and $\mathrm{CCD}_{\text {sep }}$ (9) are limited in the weak pairing regime by their common separable structure, both reducing to $\left(1+\Gamma_{P}^{\dagger} \Gamma_{H}^{\dagger}\right)|\mathrm{HF}\rangle$ at $|G| / \epsilon \ll 1$. On the other hand, the full generality of the structure matrix of the vCCD wave function (10) allows it to capture precisely all the correlations in this regime: to leading order the exact correlation energy is $E_{c \text {,exact }}=-\sum_{p h}\left(G^{2} / 2\right) /\left(\epsilon_{p}-\epsilon_{h}\right)$ and corresponds to the amplitudes $z_{p h}=(G / 2) /\left(\epsilon_{p}-\epsilon_{h}\right)$. Beyond the critical value $G_{\mathrm{cr}}$ of the HF to BCS transition, however, the vCCD ansatz quickly loses its ability to describe the stronger attractive pairing correlations, becoming indistinguishable from its separable version $\mathrm{vCCD}_{\text {sep }}$ for $G / \epsilon>1$ (see also the discussion around Fig. 4 of Ref. [8]).

The situation changes in the repulsive pairing regime with vCCD performing well while the PBCS errors increase dramatically from weak to strong coupling. Interestingly, starting at a moderate repulsive coupling the $\mathrm{CCD}_{\text {sep }} \mathrm{PBCS}$ ansatz is able to improve upon the vCCD energetics, the latter wave function being limited by its rigid exponential structure.

As indicated by the thick green line next to the horizontal axis of Fig. 1, the combined $\mathrm{vCCD}_{\text {sep }} \mathrm{PBCS}$ wave function (13) leads to very accurate energetics across all regimes with the relative errors in the correlation energy not exceeding $3 \times 10^{-4}$ for attractive pairing. Note, however, that it is essential to enable all parameters to vary freely in the minimization process as to retain the full flexibility of the wave function and thus recover all available dynamical correlations. Indeed, by considering a variational $\mathrm{CCD}_{\text {sep }}$ on top of the frozen optimal PBCS reference (or vice versa) we find significant improvements only in the weak pairing regime, as indicated by the dashed lines in Fig. 1. In particular, by varying in this way just the PBCS amplitudes within $\mathrm{vCCD}_{\text {sep }} \mathrm{PBCS}$ while keeping fixed the optimal $\mathrm{CCD}_{\text {sep }}$ structure (computed beforehand) we find no improvement over the full vCCD results for $G>G_{\mathrm{cr}}$. At $|G| / \epsilon \ll 1$, the results differ because of the limitations discussed around Eq. (14).

The relative errors for the correlation energy (29) relative to its exact value are shown in logarithmic scale in Fig. 2 for a slightly larger system of $L=20$ levels at half-filling. Note that the $\mathrm{vCCD}_{\mathrm{sep}} \mathrm{PBCS}$ errors have slightly increased with respect to the $L=12$ case, but are still respectably accurate when compared to PBCS. The limitations of the $\mathrm{vCCD}_{\text {sep }} \mathrm{PBCS}$ wave function in the weak pairing regime originate in the approximate form of its structure matrix, as remarked around Eq. (14).

This weak pairing behavior may be improved at a reasonable computational cost by including additional nonseparable CCD excitations on top of $\mathrm{vCCD}_{\text {sep }} \mathrm{PBCS}$. The $\mathrm{vCCD}_{\text {sep }}^{(w)} \mathrm{PBCS}$ ansatz (17) already provides more than an order of magnitude lower errors with respect to $\mathrm{vCCD}_{\text {sep }} \mathrm{PBCS}$ at weak pairing even for the modest $w=4$. A larger nonseparable CCD excitation window $w=6$ naturally accounts for an additional amount of correlations, further reducing the errors at weak pairing. Beyond the critical value $G_{\mathrm{cr}}$, however, there is no significant benefit of the supplementary nonseparable excitations. This is also the situation for the $\mathrm{vCCD}^{(w)} \mathrm{PBCS}$ ansatz (15) which displays the same strong pairing behavior as the plain PBCS. With the Fermi sea being washed out at 


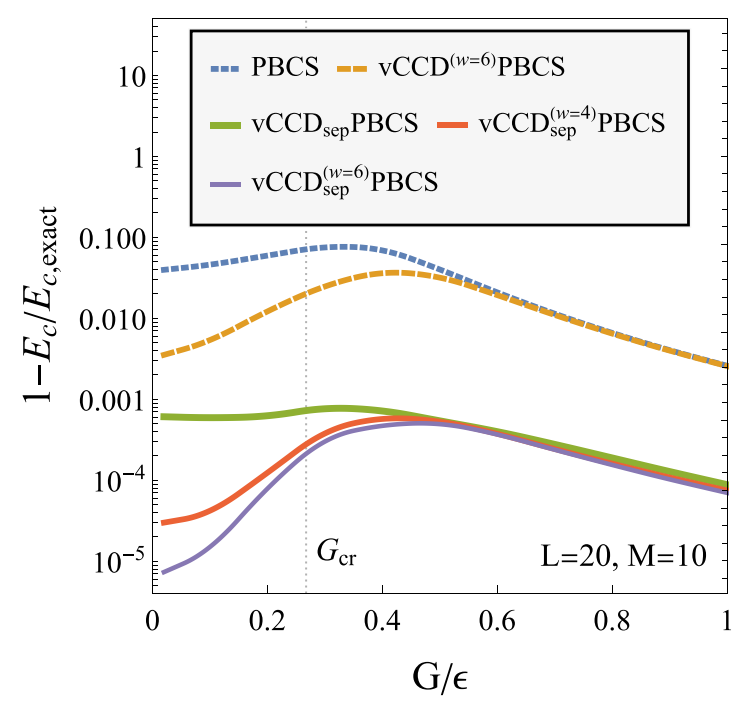

FIG. 2. Error in the correlation energy (29) relative to its exact value (in percentages) versus the pairing strength $G$ (in units of the level spacing $\epsilon$ ) for the fully variational PBCS (6), vCCD ${ }_{\text {sep }} \mathrm{PBCS}$ (13), $\mathrm{vCCD}^{(w=6)} \mathrm{PBCS}(15)$, and $\mathrm{vCCD}_{\text {sep }}^{(w=4,6)} \mathrm{PBCS}$ (17) wave functions in the case of an $L=20$ level system at half filling.

strong pairing, only a global deformation of PBCS such as $\mathrm{vCCD}_{\text {sep }} \mathrm{PBCS}$ is able to bring substantial improvement to the energetics, as opposed to any local deformation such as $\mathrm{vCCD}^{(w)}$ PBCS (15).

The numerical values for the optimal nonseparable structure matrix elements $z_{p h}$ are typically found to be very small, of the order $10^{-2}-10^{-3}$ upon the full $\mathrm{vCCD}_{\text {sep }}^{(w)} \mathrm{PBCS}$ energy minimization. In practice, within the $\mathrm{vCCD}_{\text {sep }}^{(w)} \mathrm{PBCS}$ approach we chose to limit the nonseparable CCD excitations to linear order,

$$
\operatorname{vCCD}^{(w)}(z)=1+\sum_{p=M+1}^{M+w / 2} \sum_{h=M+1-w / 2}^{M} z_{p h} P_{p}^{\dagger} P_{h}^{\dagger},
$$

which allows for a decrease in the computational complexity without any noticeable loss in precision with respect to the full form of Eq. (15).

An additional computational speed-up is enabled by limiting the action of the particle number projection operations in Eqs. (18) and (20) to a reduced subspace of particle and hole collective pairs. For the moderate values of the pairing strength $G$ considered here ( 2 to 3 times the value of $G_{c r}$ ) this still allows for an exact particle-number conservation (within the numerical accuracy). This key computational aspect is ensured by the negligible contributions of the high order terms

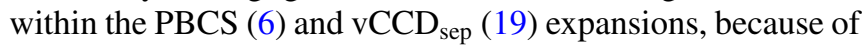
the small (subunitary) numerical values of their corresponding collective pair amplitudes. All results presented below for $L=$ 60 and $L=100$ were obtained upon projecting the particle number only within a $\mathcal{C}_{1}=15$ ph-pair subspace for PBCS in Eq. (18) and within a $\mathcal{C}_{2}=10$ ph-pair subspace for $\mathrm{CCD}_{\text {sep }}$ in Eq. (20). The gauge angles were adjusted accordingly, i.e., $\theta_{n}=2 \pi n /\left(\mathcal{C}_{1}+1\right), \phi_{k}=\varphi_{k}=2 \pi k /\left(\mathcal{C}_{2}+1\right)$. We also note that for all considered systems it was sufficient to restrict the amount of $\mathrm{CCD}_{\text {sep }}$ excitations by imposing an upper bound at $\ell_{\max }=7$ in the $\ell$ sum of Eq. (23), as to avoid the numerical errors originating from the combination of large factorials and rapidly oscillating phases and, at the same time, preserving the numerical precision.

We present in Fig. 3 the relative errors in the correlation energy (29) relative to its exact values for $L=60$ (left panel) and $L=100$ (right panel) at half filling. The
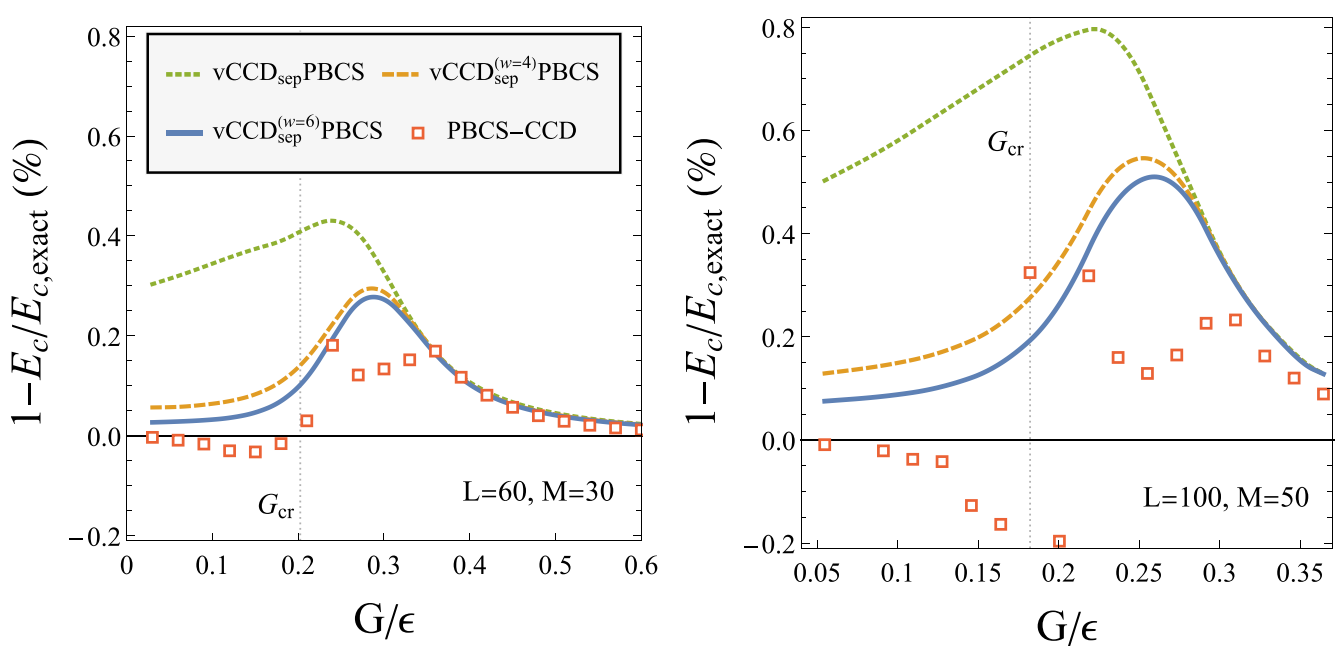

FIG. 3. Error in the correlation energy (29) relative to its exact value (in percentages) versus the pairing strength $G$ (in units of the level spacing $\epsilon$ ) for the fully variational $\mathrm{vCCD}_{\text {sep }} \mathrm{PBCS}(13)$ and $\mathrm{vCCD}_{\text {sep }}^{(w)} \mathrm{PBCS}$ (17) wave functions, in the case of an $L=60$ level system (left panel) and for an $L=100$ level system (right panel) at half filling. The red squares indicate the results of the particle-number-projected Bogoliubov-coupled-cluster theory of Ref. [18]. Note also that for $L=100$, the errors for the pure PBCS (6) reach a maximum of about $22 \%$ at $G / \epsilon=0.24$ and decrease down to $6 \%$ at very small $G$ (see also Fig. 1 of Ref. [18]). 


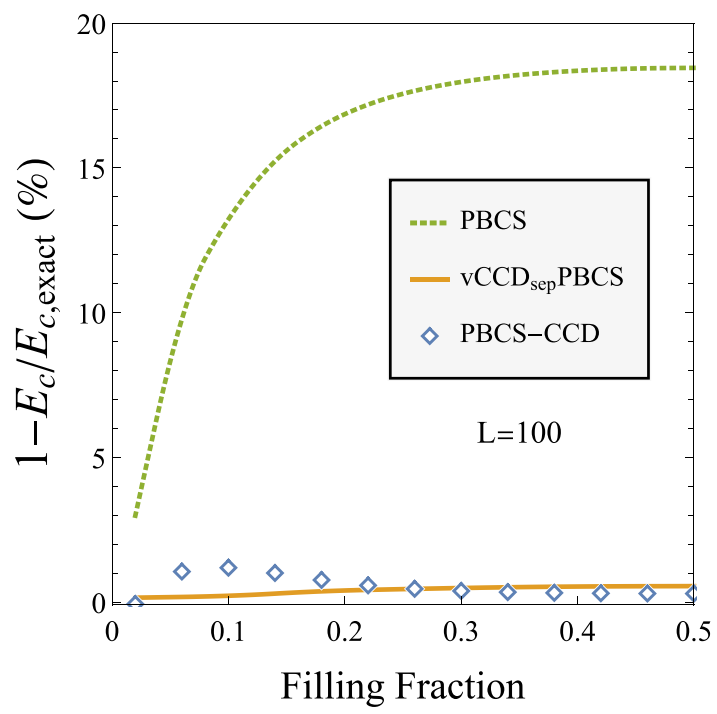

FIG. 4. Error in the correlation energy (29) relative to its exact value (in percentages) versus the filling fraction $M / L$ of an $L=100$ level system for the fully variational PBCS (6) and $\mathrm{vCCD}_{\text {sep }} \mathrm{PBCS}$ (13) wave functions. The pairing strength is $G=1.5 G_{\mathrm{cr}}$, with $G_{\mathrm{cr}}$ the critical strength at half filling. The blue diamonds indicate the results of the particle-number-projected Bogoliubov-coupled-cluster theory of Ref. [18].

$\mathrm{vCCD}_{\text {sep }} \mathrm{PBCS}$ wave function remains surprisingly accurate given that its doubly separable structure matrix (14) at weak coupling involves only $L$ independent parameters (down from a total of $2 L$ parameters because of the present ph symmetry) as compared to the fully nonseparable CCD structure matrix that requires $L^{2} / 8$ independent parameters (still for the present ph-symmetric systems). More precisely, the $\mathrm{vCCD}_{\text {sep }}$ PBCS errors at weak coupling are $0.3 \%$ and $0.5 \%$ for $L=60$ and $L=100$, respectively, increasing slightly until $G \sim G_{\mathrm{cr}}$ and then rapidly decreasing at stronger couplings.

While the inclusion of nonseparable local CCD excitations within the $\mathrm{vCCD}_{\text {sep }}^{(w)} \mathrm{PBCS}$ ansatz (17) significantly improves the error at weak coupling even for relatively very small values of $w$, perfectly accurate energetics would still require a set of global ph excitations on top of $\mathrm{vCCD}_{\text {sep }} \mathrm{PBCS}$. Nevertheless, the overall quality of the $\mathrm{vCCD}_{\text {sep }}^{(w=6)} \mathrm{PBCS}$ results is at the level of the more involved particle-numberprojected Bogoliubov-coupled-cluster theory of Ref. [18], while consistently providing an energy upper bound across all regimes.

The situation is similar away from half filling, as shown in Fig. 4. To provide a comparison with the results of Ref. [18], we consider the $L=100$ case at an interaction strength of $G=1.5 G_{\text {cr }}$. For this value the particle-number symmetry is broken for all filling fractions. Both PBCS and $\mathrm{vCCD}_{\text {sep }} \mathrm{PBCS}$ are most accurate for small (or large) filling fractions, but their errors live on different scales ( $0.5 \%$ vs $20 \%$ at half filling). As seen in Fig. 3, in the considered regime the nonseparable local $\mathrm{CCD}^{(w)}$ excitations bring no improvement over the $\mathrm{vCCD}_{\text {sep }} \mathrm{PBCS}$ results, which are well matched against those of Ref. [18].

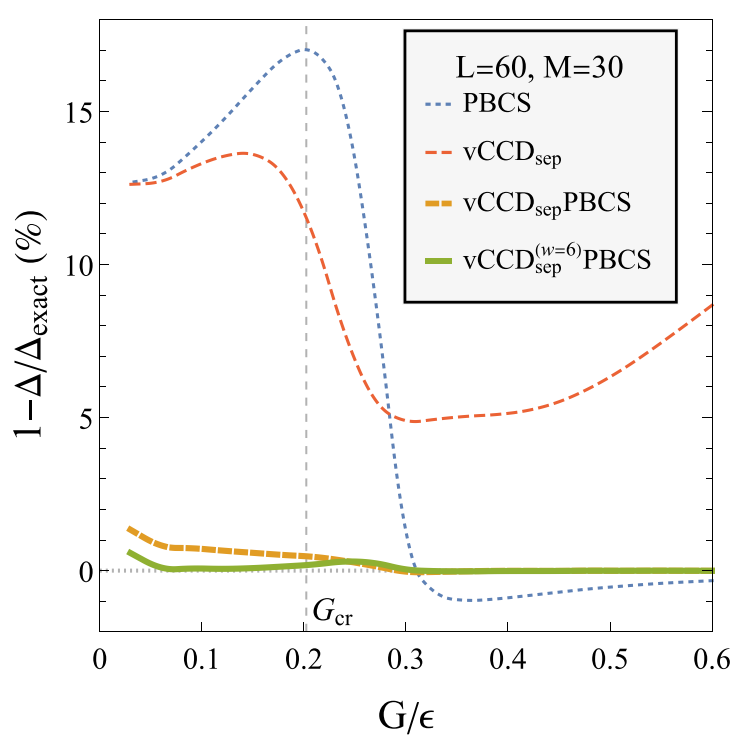

FIG. 5. Error in the canonical gap (31) relative to its exact value (in percentages) versus the pairing strength $G$ (in units of the level spacing $\epsilon$ ) for the fully variational PBCS (6), vCCD sep (9), $\mathrm{vCCD}_{\text {sep }} \mathrm{PBCS}(13)$, and $\mathrm{vCCD}_{\text {sep }}^{(w=6)} \mathrm{PBCS}(17)$ wave functions in the case of an $L=60$ level system at half filling.

Finally, we consider the behavior of the canonical gap,

$$
\Delta=G \sum_{i=1}^{L} \sqrt{n_{i}\left(1-n_{i}\right)},
$$

where $n_{i}=\left\langle c_{i}^{\dagger} c_{i}+c_{\bar{i}}^{\dagger} c_{i}\right\rangle / 2$ indicates the occupation probability of each level $i$. This quantity exhibits a more pronounced sensitivity to the structure of the wave function than the correlation energy from its dependence on the occupation probabilities.

We show in Fig. 5 the error for the canonical gap $\Delta$ relative to its exact value for $L=60$ at half filling. Improving on both individual PBCS and $\mathrm{vCCD}_{\text {sep }}$ wave functions, their combination $\mathrm{vCCD}_{\text {sep }} \mathrm{PBCS}$ only shows visible $\sim \%$ errors in the $G<G_{\text {cr }}$ region. These are further reduced within the $\mathrm{vCCD}_{\text {sep }}^{(w)} \mathrm{PBCS}$ approach which exhibits highly accurate occupations across all regimes.

\section{SUMMARY AND CONCLUDING REMARKS}

In this work, we considered a variational approach for the ground state of finite paired systems based on a combined coupled-cluster and particle-number-projected BCS wave function. We benchmarked our results against the exact solution for a picket-fence model involving a pure pairing force acting on a space of doubly degenerate, equally distanced levels. The analyzed systems range from small $(L=$ $M=12)$ to relatively large $(L=M=100)$.

We confirmed within the variational context that the combination of symmetry-restored mean-field theory and coupled-cluster theory leads to a wave function that is significantly better than either of the two taken separately, which is also the main conclusion of Ref. [18]. By incorporating pure four-body correlations, our vCCD $\mathrm{Dep}_{\text {sep }} \mathrm{PBCS}(17)$ is able to 
reproduce well the physics at weak pairing while also offering orders of magnitude smaller errors relative to the standard pair-only PBCS (6) across all other regimes. Our results match the high level of precision of the particle-number-projected Bogoliubov-coupled-cluster theory of Ref. [18], while consistently providing an energy upper bound.

Computational limitations include the restriction of the beyond-pair correlations to a relatively small window around the Fermi level (discussed in detail in the main text) and also the need for a restricted space for particle-number projection. While the latter does not spoil the exact particle-number conservation (within the numerical accuracy) for moderate values of the pairing strength, alternative approaches need to be considered for the very strong pairing regime $G \gg G_{\mathrm{cr}}$. One possibility would involve limiting the separable CCD excitations to linear order within the $\mathrm{vCCD}_{\text {sep }} \mathrm{PBCS}$ approach. Computations with this simpler wave function could then be performed efficiently without resorting to any approximations for an improvement over the already very good PBCS results for this regime.

The vCCD $\mathrm{sep}_{\text {sep }}^{(w)} \mathrm{PBCS}(17)$ wave function was shown to be quite flexible but it is still affected by structural limitations leading to a visible maximum in the energy error around $G \sim 1.5 G_{\text {cr }}$ in all analyzed cases. Attempts at mitigating these effects have included treating as independent variational parameters the factorials in Eq. (23) originating from the $\mathrm{vCCD}_{\text {sep }}$ expansion (9), with only marginal benefits.

Possible avenues to explore could involve the variational treatment of beyond-ph CCD excitations on top of the symmetry-restored mean-field state as in

$$
|\Psi\rangle=\exp \left(\sum_{i, j=1}^{L} z_{i j} c_{i}^{\dagger} c_{\bar{i}}^{\dagger} c_{\bar{j}} c_{j}\right)|\mathrm{PBCS}\rangle,
$$

which would act as a more natural choice (albeit more computationally challenging) for a regime lacking a well-defined Fermi sea. As a first step one could envision building a multiseparable beyond-ph CCD excitation operator which could be optimized with the very recently developed methods for constructing linearly independent PBCS states [19].

Finally, we leave to future studies extensions of the theory presented in this work that could incorporate the effect of seniority breaking terms within a generalized variational wave function.

\section{ACKNOWLEDGMENTS}

We thank P. Schuck for valuable discussions. This work was supported by a grant from the Romanian Ministry of Education and Research, CNCS-UEFISCDI, Project No. PNIII-P1-1.1-PD-2019-0346, within PNCDI III, and Project No. PN-19060101/2019-2022; and Spanish Ministerio de Ciencia e Innovación and European Regional Development Fund (FEDER) Project No. PGC2018-094180-B-I00. We thank the authors of Ref. [18] for sharing their numerical results with us.
[1] J. Bardeen, L. N. Cooper, and J. R. Schrieffer, Theory of Superconductivity, Phys. Rev. 108, 1175 (1957).

[2] A. Bohr, B. R. Mottelson, and D. Pines, Possible analogy between the excitation spectra of nuclei and those of the superconducting metallic state, Phys. Rev. 110, 936 (1958).

[3] K. Dietrich, H. J. Mang, and J. H. Pradal, Conservation of particle number in the nuclear pairing model, Phys. Rev. 135, B22 (1964).

[4] J. A. Sheikh and P. Ring, Symmetry projected Hartree Fock Bogoliubov equations, Nucl. Phys. A 665, 71 (2000).

[5] G. E. Scuseria, C. A. Jimenez-Hoyos, T. M. Henderson, K. Samanta, and J. K. Ellis, Projected quasiparticle theory for molecular electronic structure, J. Chem. Phys. 135, 124108 (2011).

[6] A. Khamoshi, T. M. Henderson, and G. E. Scuseria, Efficient evaluation of AGP reduced density matrices, J. Chem. Phys. 151, 184103 (2019).

[7] N. Sandulescu and G. F. Bertsch, Accuracy of BCS-based approximations for pairing in small Fermi systems, Phys. Rev. C 78, 064318 (2008).

[8] J. Dukelsky, S. Pittel, and C. Esebbag, Structure of the numberprojected BCS wave function, Phys. Rev. C 93, 034313 (2016).

[9] F. Braun and J. von Delft, Fixed-n superconductivity: The crossover from the bulk to the few-electron limit, Phys. Rev. Lett. 81, 4712 (1998).

[10] J. Dukelsky and G. Sierra, Density Matrix Renormalization Group Study of Ultrasmall Superconducting Grains, Phys. Rev. Lett. 83, 172 (1999).
[11] R. Richardson, A restricted class of exact eigenstates of the pairing-force Hamiltonian, Phys. Lett. 3, 277 (1963).

[12] G. Sierra, J. Dukelsky, G. G. Dussel, J. von Delft, and F. Braun, Exact study of the effect of level statistics in ultrasmall superconducting grains, Phys. Rev. B 61, 11890(R) (2000).

[13] J. G. Hirsch, A. Mariano, J. Dukelsky, and P. Schuck, Fully selfconsistent RPA description of the many level pairing model, Ann. Phys. 296, 187 (2002).

[14] J. Dukelsky, G. Dussel, J. Hirsch, and P. Schuck, Comparison between exact and approximate treatments of the pairing interaction for finite Fermi systems, Nucl. Phys. A 714, 63 (2003).

[15] T. M. Henderson, G. E. Scuseria, J. Dukelsky, A. Signoracci, and T. Duguet, Quasiparticle coupled cluster theory for pairing interactions, Phys. Rev. C 89, 054305 (2014).

[16] M. Degroote, T. M. Henderson, J. Zhao, J. Dukelsky, and G. E. Scuseria, Polynomial similarity transformation theory: A smooth interpolation between coupled cluster doubles and projected BCS applied to the reduced BCS Hamiltonian, Phys. Rev. B 93, 125124 (2016).

[17] A. Rubio-García, D. R. Alcoba, P. Capuzzi, and J. Dukelsky, Benchmarking the variational reduced density matrix theory in the doubly occupied configuration interaction space with integrable pairing models, J. Chem. Theory Comput. 14, 4183 (2018).

[18] Y. Qiu, T. M. Henderson, T. Duguet, and G. E. Scuseria, Particle-number projected Bogoliubov-coupled-cluster theory: Application to the pairing Hamiltonian, Phys. Rev. C 99, 044301 (2019). 
[19] R. Dutta, G. P. Chen, T. M. Henderson, and G. E. Scuseria, Construction of linearly independent non-orthogonal AGP states, J. Chem. Phys. 154, 114112 (2021).

[20] T. M. Henderson and G. E. Scuseria, Geminal-based configuration interaction, J. Chem. Phys. 151, 051101 (2019).

[21] T. M. Henderson and G. E. Scuseria, Correlating the antisymmetrized geminal power wave function, J. Chem. Phys. 153, 084111 (2020).

[22] T. M. Henderson, I. W. Bulik, and G. E. Scuseria, Pair extended coupled cluster doubles, J. Chem. Phys. 142, 214116 (2015).

[23] J. Ripoche, D. Lacroix, D. Gambacurta, J.-P. Ebran, and T. Duguet, Combining symmetry breaking and restoration with configuration interaction: A highly accurate many-body scheme applied to the pairing Hamiltonian, Phys. Rev. C 95, 014326 (2017).

[24] A. Khamoshi, G. P. Chen, T. M. Henderson, and G. E. Scuseria, Exploring non-linear correlators on AGP, J. Chem. Phys. 154, 074113 (2021).

[25] S. D. Baerdemacker, P. W. Claeys, J.-S. Caux, D. V. Neck, and P. W. Ayers, Richardson-Gaudin configuration-interaction for nuclear pairing correlations, arXiv:1712.1673.

[26] P. W. Claeys, J.-S. Caux, D. Van Neck, and S. De Baerdemacker, Variational method for integrability-breaking Richardson-Gaudin models, Phys. Rev. B 96, 155149 (2017).

[27] Y. Qiu, T. M. Henderson, J. Zhao, and G. E. Scuseria, Projected coupled cluster theory, J. Chem. Phys. 147, 064111 (2017).

[28] R. W. Richardson, Numerical study of the 8-32-particle eigenstates of the pairing Hamiltonian, Phys. Rev. 141, 949 (1966).

[29] H. Ui and G. Takeda, A class of simple hamiltonians with degenerate ground state. II a model of nuclear rotation: Spontaneous breakdown of rotation symmetry and goldstone theorem for finite dimensional system, Prog. Theor. Phys. 70, 176 (1983).

[30] T. Koma and H. Tasaki, Symmetry breaking and finite-size effects in quantum many-body systems, J. Stat. Phys. 76, 745 (1994).

[31] C. Yannouleas and U. Landman, Symmetry breaking and quantum correlations in finite systems: studies of quantum dots and ultracold Bose gases and related nuclear and chemical methods, Rep. Prog. Phys. 70, 2067 (2007).

[32] T. Papenbrock and H. A. Weidenmüller, Effective field theory for finite systems with spontaneously broken symmetry, Phys. Rev. C 89, 014334 (2014).
[33] P.-O. Löwdin, Quantum theory of many-particle systems. iii. Extension of the Hartree-Fock scheme to include degenerate systems and correlation effects, Phys. Rev. 97, 1509 (1955).

[34] I. Mayer, The spin-projected extended Hartree-Fock method, Adv. Quant. Chem. 12, 189 (1980).

[35] M. Bender, P.-H. Heenen, and P.-G. Reinhard, Self-consistent mean-field models for nuclear structure, Rev. Mod. Phys. 75, 121 (2003).

[36] K. Schmid, On the use of general symmetry-projected HartreeFock-Bogoliubov configurations in variational approaches to the nuclear many-body problem, Prog. Part. Nucl. Phys. 52, 565 (2004).

[37] C. A. Jiménez-Hoyos, T. M. Henderson, T. Tsuchimochi, and G. E. Scuseria, Projected Hartree-Fock theory, J. Chem. Phys. 136, 164109 (2012).

[38] P. Ring and P. Schuck, The Nuclear Many-Body Problem (Springer-Verlag, Berlin/Heidelberg, 1980).

[39] J.-P. Blaizot and G. Ripka, Quantum Theory of Finite Systems (The MIT Press, Cambridge, 1985).

[40] A. J. Coleman, Structure of fermion density matrices. ii. Antisymmetrized geminal powers, J. Math. Phys. 6, 1425 (1965).

[41] M. Sambataro and N. Sandulescu, Treatment of the like-particle pairing of quartets, J. Phys. G: Nucl. Part. Phys. 40, 055107 (2013).

[42] T. Duguet, Symmetry broken and restored coupled-cluster theory: I. Rotational symmetry and angular momentum, J. Phys. G: Nucl. Part. Phys. 42, 025107 (2015).

[43] T. Duguet and A. Signoracci, Symmetry broken and restored coupled-cluster theory: Ii. Global gauge symmetry and particle number, J. Phys. G: Nucl. Part. Phys. 44, 015103 (2017).

[44] T. Tsuchimochi and S. Ten-no, Bridging single- and multireference domains for electron correlation: Spin-extended coupled electron pair approximation, J. Chem. Theory Comput. 13, 1667 (2017).

[45] V. V. Baran and D. S. Delion, A quartet BCS-like theory, Phys. Lett. B 805, 135462 (2020).

[46] A. Zee, Quantum Field Theory in a Nutshell (Princeton University Press, Princeton, 2010).

[47] K. Peeters, CADABRA2: computer algebra for field theory revisited, J. Open Source Software 3, 1118 (2018).

[48] The NAG library (Numerical Algorithms Group, Oxford, United Kingdom) [www.nag.com/numeric/fl/nagdoc_latest/ html/e04/e04ucf.html]. 